\title{
OPTIMIZATION PROBLEM OF ALLOCATING LIMITED PROJECT RESOURCES WITH SEPARABLE CONSTRAINTS
}

\author{
I. A. Chub, ${ }^{a \dagger}$ M. V. Novozhylova, ${ }^{b}$ and M. N. Murin ${ }^{a}$
}

UDC 512.2

\begin{abstract}
The authors consider the mathematical model and solution method for the optimization problem of the allocation of limited resources of a project as a problem of the arrangement of rectangular objects, where objects being placed have variable metric characteristics that are subject to functional dependences. The partial quality criteria and the constraints of the feasible domain of the problem are formalized.
\end{abstract}

Keywords: optimization, allocation of limited resources, arrangement of geometrical objects.

\section{INTRODUCTION}

Problems of the optimal allocation of various limited resources such as financial, time, personnel, material, etc., arise in many practical fields. Two types of problems are distinguished:

- optimal enterprise resource planning [1] in batch production, where the time resource is not limited or this limit is not critical, i.e., continuous planning;

— investment project resource management [2], where a finite set of operations have a unique set of properties and subject to performance in strict time frame.

The importance of these problems increases because of the increasing pressure on economic entities caused by competition, stringent requirements of investors and ultimate customers, and other objective factors.

This range of problems is the focus of constant attention of experts [3-6]. Academician Glushkov initiated investigations in this field in our country, made a huge contribution to their organization, participated and supported the formation of the research areas such as network planning, enterprise resource management, and scheduling theory.

Academician Mikhalevich and his scientific school obtained the fundamental scientific results [7, 8], which underlie exact and approximate methods of the solution of optimization problems of industrial scheduling, including determination of shortest network paths.

Solution tools for limited-resource distribution problems based on fuzzy logic, soft computing, interval mathematics, etc., are presented in $[9,10]$.

An important class of problems of the second type are optimization problems of the resource management of construction-investment projects [3, 11], including engineering services reconstruction. The analysis of their practical formulations shows that such projects engage considerable capital investments, use a great amount of fixed and floating assets, and are long-term, which increases the risk of project failure under conditions of the dynamically varying environment.

The solution of these problems suggests continuing the studies on their modeling as multidimentional multicriteria problems of operations research theory [12, 13], including the development of a technique for the allowance for technological constraints and preferences of a decision-maker (DM) in forming the feasibility region of the optimization problem.

${ }^{a}$ National University of Civil Protection of Ukraine, Kharkov, Ukraine, †igorchub1959@gmail.com and chubia@apbu.edu.ua. ${ }^{b}$ National University of Construction and Architecture, Kharkov, Ukraine. Translated from Kibernetika i Sistemnyi Analiz, No. 4, July-August, 2013, pp. 173-185. Original article submitted November 15, 2012. 


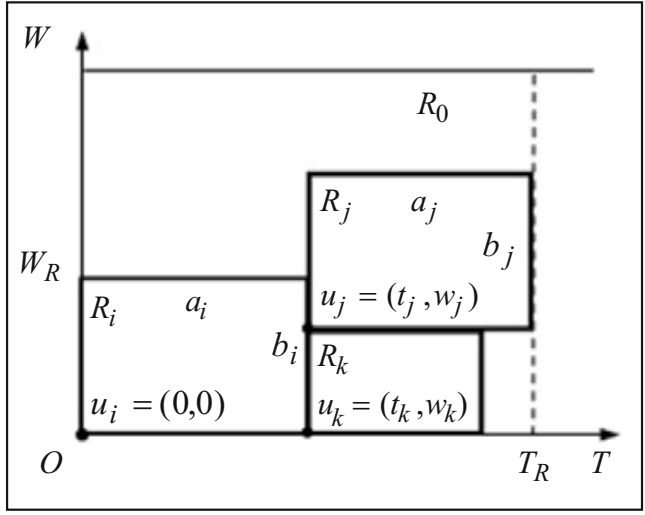

Fig. 1. Schematic arrangement of jobs $\left\{R_{i}, R_{j}, R_{k}\right\}$ in resource space.

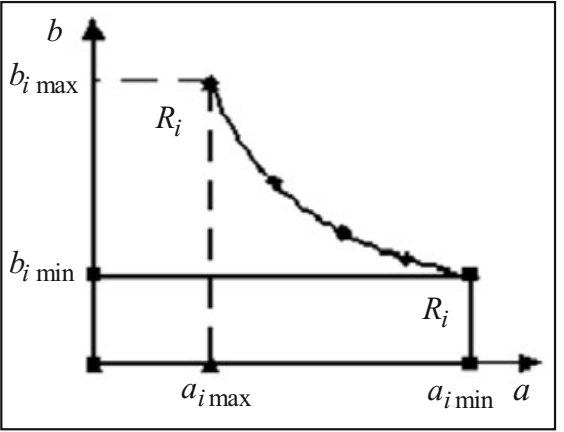

Fig. 2. Graphic representation of job $R_{i}$ for different metric characteristics.

In the paper, we will develop the approach proposed in [14, 15] and based on the use of results of the field of optimization geometrical design theory [16] such as the arrangement of geometrical objects with variable metric characteristics and spatial shape in a bounded domain [17].

The purpose of the paper is to determine and formalize the partial criteria of solution quality and the set of constraints for the feasibility region of the optimization problem of the allocation of limited resources of the project as the problem of the arrangement of rectangular objects with variable metric characteristics, related by functional dependences, development of optimization methods of problem solution, and carrying out numerical experiments.

\section{PROBLEM STATEMENT AND CONSTRUCTION OF THE OPERATION MODEL}

Let us consider the practical resource management problems arising at different stages of the construction-investment project.

Let there be a project $R$ consisting of $N$ jobs (operations), $R=\left\{R_{i}\right\}, i=1, \ldots, N$. The partial order condition $R_{i} \prec R_{j}$, $i, j \in\{1, \ldots, N\}, i \neq j$, defined by the specific sequence of jobs (job $R_{j}$ immediately follows $R_{i}$ ) is specified on the set of jobs $R$. Note that determining this sequence involves the DM (project manager) since in practice there can be several such sequences.

For each job $R_{i}$ its amount $S_{i}$ expressed in man $\cdot$ hours, $S_{i}=$ const is known. No more than $W_{i}$ immediate executors are allocated for the project in the large at every instant of time.

It is necessary to make a schedule of project jobs, optimal in the required resources.

Let us consider this problem as a $2 D$ problem of the theory of optimizational geometrical design, within which the properties of the objects under study are interpreted as geometrical characteristics. Then project resources in the large can be represented as a domain $R_{0}$ of the two-dimensional space of resources $O T W$, where $O T$ is the time axis (project runtime), $O W$ is the axis of the work force whose scale is coordinated with measure units of $T$.

Each job $R_{i}$ of the project $R$ can be represented as a layout object $R_{i}$, of rectangular shape $R_{i}\left(a_{i}, b_{i}\right)$. The metric characteristics $a_{i}$ and $b_{i}$ denote the job run time $R_{i}$ and the number of its executors, respectively, at each instant of time. The time of the beginning of job execution $R_{i}$ and its association with the necessary amount of work force are determined by the parameters $v_{i}=\left(t_{i}, w_{i}\right)$ of job arrangement in the resource space OTW (Fig. 1).

Remark 1. On the assumption that metric characteristics of the objects $R_{i}, i=1,2, \ldots, N$, are variables: $a_{i}, b_{i}-$ var, and the volume $S_{i}, S_{i}=a_{i} \times b_{i}=$ const, the relation $b_{i}=S_{i} / a_{i}$ holds.

Remark 2. Based on the allowance for the technical characteristics of the project for executing each job $R_{i}$, the maximum and minimum admissible values of resources are chosen. In other words, metric characteristics of object $R_{i}$ are elements of sets $A$ and $B$ (Fig. 2):

$$
a_{i} \in A_{i}, b_{i} \in B_{i}
$$

where $A_{i}=\left[a_{i \min }, a_{i \max }\right], B_{i}=\left[b_{i \min }, b_{i \max }\right], a_{i \min }>0$, and $b_{i \min }>0$. 
Depending on the formulation of a specific problem, the sets $A$ and $B$ can be both continuous and discrete. If resource representation is discrete,

$$
b_{i}=\left\lceil S_{i} / a_{i}\right\rceil .
$$

Note that the relations $S_{i} \leq a_{i \min } \times b_{i \max }$ and $S_{i} \leq a_{i \max } \times b_{i \min }$ hold for $\left\lceil S_{i} / a_{i}\right\rceil \neq S_{i} / a_{i}$. Hence, in such formulation parameters $u_{i}=\left(v_{i}, a_{i}\right)=\left(t_{i}, w_{i}, a_{i}\right)$ are endogenic for job $R_{i}$. Next we assume that the notation $R_{i}$ is equivalent to $R_{i}\left(u_{i}\right)$.

The set of jobs $R_{j}$ immediately following $R_{i}$ can consist of more than one element $j \in\{1,2, \ldots, J\}, 1 \leq j<N, i \neq j$. Denote by $I_{i}^{j}$ the set of indices of such jobs.

Thus, the optimal resource allocation problem can be formulated as the problem of the arrangement of $N$ rectangles $\left\{R_{i}\right\}$ without overlapping in a rectangle $R_{0}$ of the form

$$
W_{R} \times T_{R} \rightarrow \min _{\mu \in D \subset E^{3 N+2}}
$$

where $W_{R}$ and $T_{R}$ are metric characteristics of the rectangular layout domain $R_{0}, u=\left(u_{1}, u_{2}, \ldots, u_{N}\right)$ and $D$ is the feasibility domain. It has the following system of constraints:

- layout of the set of objects $R$ in $R_{0}$ (the condition of the presence of each job as a part of the project)

$$
R_{i} \subset R_{0} ;
$$

— the condition of mutual pairwise non-intersection of the objects (ensuring the impossibility of using one resource by two jobs simultaneously)

$$
\text { int } R_{i} \cap \text { int } R_{j}=\varnothing \text {; }
$$

— the condition of partial order of the jobs

$$
R_{j} \succ R_{i}, j \in I_{i}^{j}
$$

— constraints for the value of job resources

$$
a_{i} \in A_{i}, b_{i} \in B_{i} ;
$$

— the condition of retaining the amount of job of the project

$$
b_{i}=S_{i} / a_{i}, i, j=1,2, \ldots, N, i \neq j .
$$

In view of Remark 1, the problem under study belongs to the class of problems of the arrangement of geometric objects with variable metric characteristics provided that the latter are related by functional dependences.

As a matter of fact (and by the standard solution technique [13]), the problem is two-criteria and can be represented as a sequence of two one-criterion problems:

$$
\begin{gathered}
T_{R} \rightarrow \min _{u \in D_{1} \subset E^{2 N+1}}, \\
W_{R} \rightarrow \min _{u \in D_{2} \subset E^{2 K+1}} .
\end{gathered}
$$

The subdomain $D_{1}$ of the feasible domain $D$ of problem (9) is defined without constraints for $W_{R}$ (or $W_{R}$ is assumed sufficiently large).

Solving problem (9) yields the duration $T_{R}^{*}$ of the critical path [13] of the project $R$, the set of critical operations $R_{k}$, $k=1,2, \ldots, K_{1}$, whose total duration constitutes the duration $T_{R}^{*}$ of the critical path, and total time reserves for noncritical operations $\bar{R}_{k}, k=1,2, \ldots, K, K+K_{1}=N$.

The domain $D_{2}$ of the feasible solutions of problem (10) is defined by the constraints (4)-(8) provided that $T_{R}=T_{R}^{*}$ and the parameters of the arrangement of objects modeling critical operations $R_{k}, k=1,2, \ldots, K_{1}$, are constant.

In solving problems (9), (10), parameters $a_{i}, i=1,2, \ldots, N$, of the objects are fixed (generally speaking, for problem (10) this condition is obligatory only for critical operations). Criterion (10) can be represented as 


$$
\Delta W \rightarrow \min _{u \in D_{2} \subset E^{2 K+1}}
$$

where

$$
\Delta W=\max _{t \in\left\{1,2, \ldots, T_{R}\right\}} W_{t}-W_{t-1},
$$

which is an equivalent representation in terms of the basic problem.

Thus, the ultimate goal of the solution of problem (3)-(8) is to construct the optimal schedule $G\left(T_{R}, \Delta W\right)$ of the project $R$.

If the parameters of the project obtained as a result of the solution of problems (9), (11), and (12) are such that additional resources can be allocated for its prompt execution, then the third problem occurs, of bringing the project to a more intensive level of performance [13] of the form

$$
T_{R} \rightarrow \min _{u \in D_{3} \subset E^{Z}},
$$

where $Z=3 K_{1}+2 K+1$. The metric characteristics of the objects modeling the critical operations $R_{k}, k=1,2, \ldots, K_{1}$, are variable.

\section{ANALYTIC DESCRIPTION OF CONSTRAINTS (4)-(6)}

The condition of the arrangement (4) of the set of objects $R_{i}$ in the domain $R_{0}$ with allowance for Remarks 1 and 2 and expression (7) is specified by the system of linear inequalities

$$
\left\{\begin{array}{l}
t_{i} \geq 0 \\
T_{R}-t_{i}-a_{i} \geq 0 \\
w_{i} \geq 0, \\
W-w_{i}-\frac{S_{i}}{a_{i}} \geq 0, \\
-a_{i}+a_{i \max } \geq 0 \\
a_{i}-a_{i \min } \geq 0
\end{array} \quad i=1, \ldots, N .\right.
$$

The mutual non-intersection condition (5) is specified by the set of linear inequalities

$$
\left(\begin{array}{l}
t_{j}-t_{i}-a_{i} \geq 0 \\
w_{j}-w_{i}-S_{i} / a_{i} \geq 0, \\
t_{i}-t_{j}-a_{j} \geq 0, \\
w_{i}-w_{j}-S_{j} / a_{j} \geq 0
\end{array} \quad i, j=1, \ldots, N, i \neq j .\right.
$$

The partial ordering condition (6) is represented as a system of linear inequalities

$$
t_{j}-t_{i}-a_{i} \geq 0, j \in I_{i}^{j}, i, j=1,2, \ldots, N, i \neq j
$$

Two approaches are possible in this case (proceeding from the scheduling practice). If the condition that job $R_{j}$ follows immediately after job $R_{i}$ is obligatory, then the respective constraint in (16) is a strict equality. Otherwise condition (16) means that job $R_{j}$ should be executed not earlier than job $R_{i}$ is completed. Next it is shown that the presence or absence of condition (16) and of its more stringent analog in the form of equality considerably influences the estimate of the computation complexity of the problem solution algorithm.

Let constructing the schedule $G\left(T_{R}, \Delta W\right)$ of project $R$ necessarily imply the solution of problem (13). Considering this condition, we will present the main properties of the optimization problem (3)-(8).

Property $D$ _ 1. The domain $D$ is a nonconvex disconnected bounded point set with piecewise smooth boundary $\Psi=$ Fr $D$. Each connectivity component of the feasible domain $D$ is multiply connected.

Property D_2. The number of constraints for the feasible domain $D$ of problem (3)-(8) quadratically depends on the number of the objects. 
Property D_3. Domain $D$ is representable as a union of a finite number of subdomains $D_{g}$ of the form

$$
D=\bigcup_{g=1}^{G} D_{g}, G=\mathrm{O}\left(4^{N(N-1) / 2}\right)
$$

The subdomain $D_{g}$ is described by the system $F_{g}(u) \geq 0$, which includes $N$ systems of nonlinear inequalities (14), $N(N-1) / 2$ inequalities (one from each set of inequalities (15) for each pair of objects), and the partial ordering condition (16).

Property D_4. Functions of the form $f\left(t_{i}, t_{j}, a_{j}\right)=t_{i}-t_{j}-\frac{S_{j}}{a_{j}}$ and $f\left(t_{i}, t_{j}, a_{j}\right)=t_{j}-t_{i}-\frac{S_{i}}{a_{i}}$ are convex and belong to the class of separable functions [18].

Property D_5. Objective functions of the considered partial problems (9), (10), (13) are linear. Hence, the optimal solution of these problems is reached on the boundary of the appropriate sets $D_{1}, D_{2}, D_{3}$.

Property D_6. In the general case, the optimal solution $u^{*}$ of each partial problem is determined by the system $F^{*}(u)=0$ of linear (and nonlinear for problem (13)) equations from $F_{g}(u) \geq 0$. The rank $I$ of system $F^{*}(u)=0$ is equal to the dimension of the space where the problem is considered. The system constraints $F^{*}(u)=0$ are called job list [18].

This property is not obvious for problem (13), but it holds since the convex functions of the nonlinear constraints are single-valued by formulation.

Property D_7. For any point $u^{*}$ the relation $u^{*} \in \bigcap_{q>1} D_{g q}$ holds.

The constraints of the problem that are transformed to equalities at the current point $u$ are called active set [18]. From the property $D \_7$ it follows that for the considered problems the dimension $J$ of the active set is greater than $I$.

\section{SOLUTION METHODS FOR PROBLEM (3)-(8)}

In view of the properties $D_{-} 1$ and $D_{-} 3$ of the feasible domain, the optimization problem under study as well as its partial subproblems (9), (10), and (13) are a multidimentional multiextremum combinatorial optimization problem with disconnected feasible domain belonging to the class of NP-hard problems [20].

The tools of the solution of partial problems (9), (10) (provided that the metric characteristics of the objects are constant) as rectangular arrangement problems with a linear objective function and linear constraints are adequately represented in the scientific literature, for example in [21, 22]. Therefore, in the present paper we consider the modifications of exact and approximate methods of local and global optimization for the solution of problem (13).

Exact Solution Methods for Problem (13). According to [18], it is theoretically possible to determine the global minimum of the objective function of problem (13).

Based on the characteristics of the constraint functions of the problem and property $D \_3$ of the feasible domain, problem (13) belongs to the class of nonlinear combinatorial optimization problems. The general ideology of the solution of such problems is to construct a solution tree (we call it $A$ ) and use it to order the exhaustive search of subsets of the feasible domain of the problem, with a more simple structure, and to determine the locally optimal solution on each such subset.

Two implementations of the solution tree $A$ are possible: the first is a tree $A^{1}$ constructed based on the exhaustive search of the convex subsets $D_{g}$ of the feasible domain of the problem (property $D_{-} 3$ ), the second is a tree $A^{2}$ constructed based on the set of systems of equations containing the system $F^{*}(\mu)=0$ (property $D_{-} 6$ ).

Let us consider the special features of the modifications of both approaches to the formation of the structure of the solution tree of problem (13) taking into account variable metric characteristics of the objects and the partial ordering condition on the set of objects.

Implementation 1. The root of the solution tree $A_{0}^{1}$ corresponds to the system of inequalities (14), one of the four constraints from the set (15) can be added to node $A_{p(i, j)}^{1}$ at each next level, where $p(i, j)$ is the number of the pair $(i, j)$, 
$i<j$. At the last, $(N(N-1) / 2)$ th level of the tree $A^{1}$, all the convex subsets $D_{g}$ will be constructed (property $D_{-} 3$ ). Thus, the problem of searching for the global extremum reduces to the truncated enumeration and solution of a finite set of convex programming problems with linear objective function.

The upper-bound estimate of the number of nodes at the last level of the solution tree $A^{1}$ is equal to $G$ from (17), and the variability of the metric characteristics of the rectangles $R_{i}$, which model critical operations, does not influence the number of tree nodes. However, (17) is an overestimate since allowance for constraint (16), especially in the form of an equality, reduces the number of the considered nodes of the solution tree for each specific implementation of the problem.

Assume that the partial order condition has the form of the equality

$$
t_{j}-t_{i}-a_{i}=0, j \in I_{i}^{j}, i, j=1,2, \ldots, N, i<j .
$$

Denote $\aleph=\sum_{i=1}^{N-1} I_{i}^{j}$. Then with allowance for $(18)$ tree $A^{1}$ will have $(N(N-1) / 2-\aleph)$ levels. Moreover, the choice of the first or third node at each $p(h, j)$ th level of the solution tree is uniquely determined by a similar choice at the $p(h, i)$ th level $h<(i, j)$. The optimal problem solution on the system of inequalities $F_{g}(\mu) \geq 0$ is a local minimum of problem (13).

Implementation 2. If the metric characteristics of the rectangles (project jobs) are constant (problem (10)), the solution tree $A^{2}$ is based on the possibility of constructing a bijection $\Psi$ on the system of equations $F^{*}(v)=0$ (property $\left.D \_6\right)$ of the form

$$
\begin{gathered}
\Psi: t_{i} \leftrightarrow F_{k}^{*}(v)=0, F_{k}^{*}(v) \in\left\{t_{i}-t_{l}-a_{l}, t_{i}\right\} ; \\
\Psi: w_{i} \leftrightarrow F_{k}^{*}(v)=0, \quad F_{k}^{*} \in\left\{w_{i}-w_{l}-b_{l}, w_{i}\right\} ; \\
\Psi: T_{R} \leftrightarrow F_{k}^{*}(v)=0, F_{k}^{*}(v)=T_{R}-t_{l}-a_{l}, \\
k \in\{1,2, \ldots, 2 N+1\} ; i, l=1,2, \ldots, N ; i \neq l .
\end{gathered}
$$

The solution tree $A^{2}$ is used to implement all the possible systems of equations with property (19), including the system $F^{*}(v)=0$. There are $2 N$ levels of the tree $A^{2}$, each corresponding to some variable arrangement parameter $t_{i}$ or $w_{i}$, $i=1,2, \ldots, N$. At each next level, node $A_{r(i)}^{2}, r(i)=\left\{i * 2, i^{*} 2+1\right\}$, can be supplemented with one of the two appropriate constraints from set (15), which becomes active; there are $(N-1)$ such constraints. The upper estimate of the number of nodes at the last, $2 N$ th, tree level $A^{2}$ for constant metric characteristics of the objects is equal to $(2 N)^{N}$.

If the metric characteristics $\left(a_{i}, b_{i}\right)(1)$, (2) of the object $R_{i}$ are variable, it becomes impossible to construct a bijection (19). In other words, this means that not only the number of levels of the tree $A^{2}$ increases to the $(Z+1)$ since it is now necessary to consider the variables $a_{i}$ and $T_{R}$, but also the numbers of tree nodes that can be added at the current level. The upper-bound estimate of the number of nodes is equal to $Z^{Z}$. Allowance for constraint (16) reduces the number of the considered nodes of the solution tree for each specific implementation of the problem; however, the total complexity of the problem remains nonpolynimial.

Approximate Problem Solution Methods. The approach based on the optimization with respect to groups of variables is one of the most interesting and includes two stages.

Stage 1. Determining a locally optimal solution based on the modified method of the optimization based on groups of variables.

Stage 2. The enumeration of local extrema based on redetermining the sequence of object arrangement.

In the present paper, we assume that the object allocation order is given, the more so that for problems (10), (13) the sequence of object allocation is defined by the solution of problem (9).

Let us present the framework of Stage 1 (optimization with respect to groups of variables [16]):

- the objects are allocated one by one according to a given sequence of numbers, the objects allocated earlier are considered fixed, the objects subject to the arrangement at the later iterations of the method are not taken into account; function $\Xi$.

- the current object $R_{i}$ is allocated with regard for the requirement of the minimization of the current objective 
Thus, in the general case, for the set of the considered problems at each ith iteration of the method of optimization with respect to groups of variables, a problem $\Xi \rightarrow \min _{\left(t_{i}, w_{i}, a_{i}\right) \in \Theta_{i}}$ is solved, where the domain $\Theta_{i}$ is a three-dimensional section of the subdomain $\widehat{\Theta} \subset E^{3 i}$ of feasible solutions $D$ of the main problem, $t_{l}, w_{l}, a_{l}=$ const, $l=1, \ldots, i-1$, and $t_{i}, w_{i}, a_{i}$ are var, the domain $\widehat{\Theta}$ is generated by constraints (4)-(6) for the set of objects $\left\{R_{l}\right\}, l=\overline{1, i}$.

\section{A PRIORI LINEARIZATION OF THE CONSTRAINTS}

A technique for the global linearization of nonlinear constraints of the problem is proposed in [18]. It allows linear approximation of the problem with any prescribed accuracy without increasing the dimension of the space of parameters to which the feasible domain of the problem belongs, on the assumption that variable metric characteristics of jobs are continuous.

The considered transformation $D \stackrel{\Im}{\rightarrow} D^{L}$ replaces the nonlinear constraint functions from (14) and (15) with the appropriate linear functions (one or several).

The number of approximation elements depends on its accuracy $\varepsilon=\max \left(d^{n}-d_{k}^{n}\right)$ specified a priori, where $n$ is the number of approximation nodes, $d^{n}$ and $d_{k}^{n}$, respectively, are the coefficients of the equation of the secant connecting the adjacent nodes of the approximation, and of the equation of a tangent to the current segment of the nonlinear function.

The convexity of the nonlinear constraint function (property $D_{-} 4$ ) means the following: if the approximation accuracy $\varepsilon$ is such that more than one linear approximating function is necessary, then the linearized description of the corresponding boundary section $\Psi=\operatorname{Fr} D$ is specified by the system of the constructed linear constraints.

Thus, the mapping $\&$ for $n=1$ has the form

$$
\mathfrak{s}\left(a_{i}\left(w_{j}-w_{i}\right)+S_{i}\right)=\alpha_{i} \times a_{i}+\beta_{i} \times\left(w_{j}-w_{i}\right)-d_{i},
$$

where $\alpha_{i}=A_{i} / \delta_{i}, \beta_{i}=B_{i} / \delta_{i}, \delta_{i}=\sqrt{A_{i}^{2}+B_{i}^{2}}, A_{i}=b_{i \max }-b_{i \min }, B_{i}=a_{i \max }-a_{i \min }$, and $d_{i j}^{n}=b_{i \min } B-a_{i \max } A$.

The approximation $\mathfrak{s}\left(W-w_{i}+S_{i} / a_{i}\right)$ of the nonlinear constraint function from (14) is carried out similarly.

Let us consider the main properties of the mapping $\mathfrak{\Im}$.

Property $\mathfrak{s}$ 1. Due to the transformation $\mathfrak{\Im}$ the polyhedral approximation set $D^{L}$ belongs to the space of the same dimension as the original domain $D$. In turn, due to the transformation $D_{g} \stackrel{\Im}{\rightarrow} D_{g}^{L}$ the convex linear subdomain $D_{g}^{L}$ belongs to the same space as the original subdomain $D_{g}$ does.

Property $\mathfrak{I}_{-}$2. A single application of this transformation $(n=1)$ does not increase the number of problem constraints. The linear approximation of the set (15) of nonlinear constraints becomes

$$
\left(\begin{array}{l}
t_{j}-t_{i}-a_{i} \geq 0, \\
\alpha_{i} a_{i}+\beta_{i} \times\left(w_{j}-w_{i}\right)-d_{i j} \geq 0, \\
t_{i}-t_{j}-a_{j} \geq 0, \\
\alpha_{j} a_{i}+\beta_{j}\left(w_{i}-w_{j}\right)-d_{i j} \geq 0
\end{array} i, j=1, \ldots, N, i \neq j .\right.
$$

Property $\mathfrak{3}$ 3. Multiple application of the approximating procedures increases the number of constraints (20) but does not change the number $G$ of subsets $D_{g}$. Hence, such characteristics of the solution tree $A^{1}$ applied to order the subsets $D_{g}$ in order to obtain the globally optimal solution also remain invariable, as the number of levels of the solution tree and the number of nodes of the tree added at each intermediate level. 


\section{MODIFICATION OF THE METHOD OF LOCAL OPTIMIZATION OF LINEARIZED PROBLEM}

A linearized problem on the subset $D_{g}^{L}$ has the form

$$
\Xi \rightarrow \min _{D_{g}^{L} \subset E^{Z}},
$$

where the domain $D_{g}^{L}$ is specified by the system $F_{g}^{L}(u) \geq \delta^{n}$ of linear constraints, components of the vector $\delta^{n}$ are zero, and the values $d_{i j}^{\eta}, d_{j i}^{\eta}, \eta=1, \ldots, n$.

Problem (21) is solved based on the active set method [18]. Let us consider the implementation of the scheme of active set, whose $(m+1)$ th iteration has the form $u^{m+1}=u^{m}+\Delta u^{m} \cdot p^{m}$, where $u^{m}$ are the values of the variables at previous iteration, $\Delta u^{m}$ is step, and $p^{m}$ is descent direction.

At the point $u^{m}$ the signs of components of the vector of Lagrange multipliers $\lambda$ are defined as solutions of the nondegenerate system of linear equations $H^{T} \lambda=c$, where $H$ is the matrix of the coefficients of job list constraints at the considered point, $c$ is the vector of coefficients of the objective function, in this case it has the form $(0,0, \ldots, 0,1)$.

In view of the features of vector $c$, the vector of Lagrange multipliers $\lambda$ is the Zth column of the matrix $H^{T}$.

If all the components $\lambda_{s} \geq 0, s=1, \ldots, Z$, then $u^{m}$ is a solution of problem (21). If some $\lambda_{s}<0$, then the direction $p^{m}$ is determined as a solution of the linear system of equations $H p^{m}=e_{S}$, where $e_{S}$ denotes the sth column of the unit matrix, i.e., $p$ is the sth column of the matrix $H^{-1}$ (the sth row of the matrix $\left(H^{T}\right)^{-1}$ ).

For the set $V$ of all constraints of problem (21) out of the job list, the upper estimate $\bar{\alpha}$

$$
\bar{\alpha}= \begin{cases}\min _{v} \frac{d_{v}^{n}-h_{v}^{T} u^{m}}{h_{v}^{T} p^{m}} & \text { if } h_{v}^{T} p^{m}<0, v \in V, \\ +\infty, & \text { if } h_{v}^{T} p^{m} \geq 0 \text { for all } v \in V,\end{cases}
$$

of the step $\Delta u^{m}$ implementing the equality $h_{v}^{T}\left(u^{m}+\bar{\alpha}^{m} p^{m}\right)=d_{v}^{n}$ is calculated at the $m$ th iteration of the method, where $v$ is the index of inactive constraint.

The presence of property $D_{-} 7$ means that vector $u^{m}$ can simultaneously satisfy two inequalities, for example, the first and the second, from set (20). In that case the inactive constraint is that with the greater estimate of the step $\Delta u^{m}$. Thus, the passage from one convex subdomain $D_{g}^{L}$ of the linearized feasible domain $D^{L}$ to the other, $D_{g+1}^{L}$, is carried out.

In other words, the constraint is selected, which will be included in the job list at the next iteration of the solution algorithm with simultaneous passage to the other convex subdomain, i.e., the motion generally takes place in a nonconvex domain.

The program was coded in Object Pascal 6.0 in the Borland Delphi 7.0 environment. The program includes (i) a data input module that is capable of setting and correcting work characteristics and sequence; (ii) an analytic module that has procedures of constructing approximation functions with a prescribed accuracy and implementing exact and approximate methods; and (iii) a data display and storage module.

The proposed tools for modeling and solution of the limited resource allocation problem were applied to solve the following practical problem. The object under study was attached warehouses with an automatic system for collecting and palletizing boxes of finished goods of the "Philip Morris Ukraine" Joint-Stock Company Kharkov. The project implied installation of an automatic fire alarm system.

Without going into details, the system is mounted and adjusted as follows:

- preparatory work (scaffolding, preparation of cables, equipment, and workplaces);

- laying of vinyl pipes and cables;

— testing of joints (for reliability of fixation of pipes on supports);

— installation of equipment and instruments.

The fire alarm system is commissioned in compliance with appropriate regulations. 
TABLE 1

\begin{tabular}{|c|c|c|c|c|c|c|}
\hline \multirow{2}{*}{$\begin{array}{c}\text { Work } \\
\text { number }\end{array}$} & \multirow{2}{*}{ Work type and materials, units } & \multirow{2}{*}{$\begin{array}{l}\text { Amount of } \\
\text { work and } \\
\text { materials }\end{array}$} & \multicolumn{2}{|c|}{$\begin{array}{l}\text { Number } \\
\text { of workers }\end{array}$} & \multicolumn{2}{|c|}{ Labor input, man $h$} \\
\hline & & & $\min$ & $\max$ & $\begin{array}{c}\text { per work } \\
\text { unit }\end{array}$ & total \\
\hline 1 & Preparatory work & 4 & 2 & 4 & 20 & 80 \\
\hline 2 & Installation of base hub, piece & 3 & 3 & 6 & 73 & 219 \\
\hline 3 & Installation of control instrument on loop 1, piece & 19 & 4 & 6 & 11.52 & 218.88 \\
\hline 4 & Installation of final control unit, piece & 5 & 4 & 6 & 50 & 250 \\
\hline 5 & Installation of control panels, piece & 1 & 1 & 2 & 34.56 & 34.56 \\
\hline 6 & Installation of power switchers, piece & 1 & 1 & 2 & 23.04 & 23.04 \\
\hline 7 & Installation of panel or indicator for up to 10 signals, piece & 2 & 1 & 2 & 13.44 & 26.88 \\
\hline 8 & Installation of control instrument for 4 loops, piece & 20 & 4 & 10 & 21.12 & 422.4 \\
\hline 9 & Installation of control instrument for 8 loops, piece & 8 & 2 & 8 & 21.12 & 168.96 \\
\hline 10 & Installation of interior tubular scaffold, piece & 1 & 2 & 5 & 70 & 70 \\
\hline 11 & Punching of circular $25 \mathrm{~mm}$ holes, piece & 100 & 2 & 5 & 0.191 & 191 \\
\hline 12 & Laying cables in pipes, $\mathrm{m}$ & 98 & 6 & 10 & 9.60 & 940.8 \\
\hline 13 & Installation of grounding bridge, piece & 5 & 1 & 1 & 0.6 & 3 \\
\hline 14 & Laying of cables in trays, $\mathrm{m}$ & 57.7 & 3 & 8 & 6.05 & 349 \\
\hline 15 & Laying of vinyl pipes, m & 98 & 6 & 20 & 27.84 & 2728 \\
\hline 16 & Penetration of wall for $27 \mathrm{~mm}$ pipes, piece & 10 & 1 & 2 & 1.07 & 10.7 \\
\hline 17 & Installation of cable boxes, piece & 10 & 1 & 2 & 0.96 & 9.6 \\
\hline 18 & Connection of single-pair cable, piece & 5 & 2 & 4 & 9.60 & 48 \\
\hline 19 & Brining out of single-pair cable, piece & 2 & 1 & 2 & 9.60 & 19.2 \\
\hline 20 & Installation of automatic alarm indicators, piece & 564 & 10 & 20 & 4.22 & 2380 \\
\hline 21 & Installation of explosion-protected alarm indicators, piece & 17 & 3 & 6 & 4.22 & 71.74 \\
\hline 22 & Installation of manual fire alarms, piece & 41 & 3 & 6 & 1.92 & 78.72 \\
\hline 23 & Mounting of light indication units, piece & 2 & 1 & 1 & 1.92 & 3.84 \\
\hline 24 & Installation of buttons on control panels, piece & 20 & 1 & 2 & 1.92 & 38.4 \\
\hline 25 & Installation of wall cabinet, piece & 19 & 2 & 4 & 3.84 & 72.96 \\
\hline 26 & Installation of $1 \mathrm{~kW}$ horn, piece & 53 & 2 & 5 & 2.88 & 152.64 \\
\hline 27 & Installation of wall light pointers, piece & 100 & 3 & 5 & 0.83 & 83 \\
\hline 28 & Routing of cables into cable boxes, piece & 172 & 1 & 2 & 0.10 & 17.2 \\
\hline 29 & Disassembling of internal tubular scaffold, piece & 1 & 2 & 5 & 70 & 70 \\
\hline 30 & Testing of alarm circuit, piece & 1 & 2 & 4 & 151.68 & 151.68 \\
\hline 31 & Commissioning of system & 1 & 1 & 1 & 24 & 24 \\
\hline
\end{tabular}

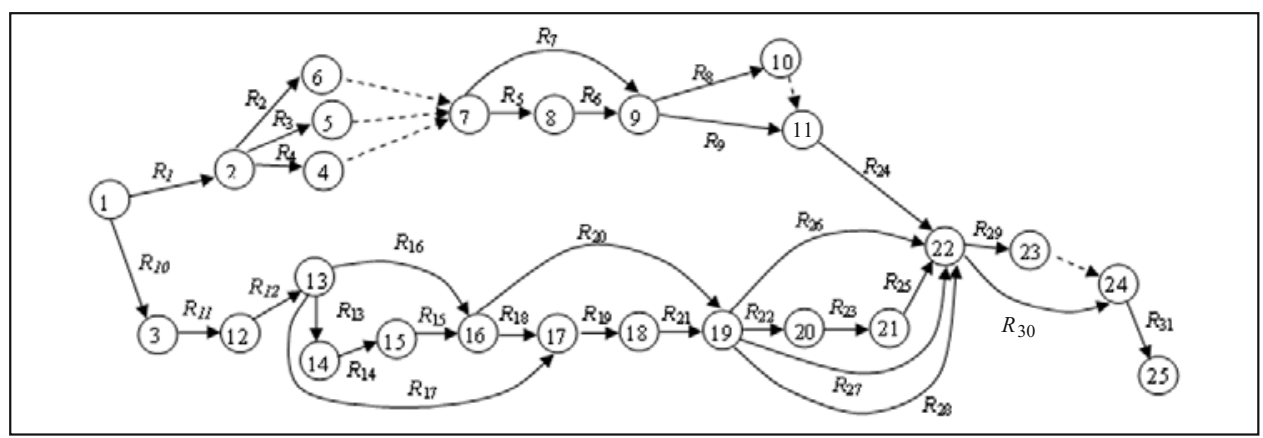

Fig. 3. Network model of works done to mount and adjustment the fire alarm system.

The total estimated cost of the project is 584.925 thousand UAH. Estimated labor input is 9.036 thousand man.h. Estimated salary is 147.613 thousand UAH. Average job class is 3.3.

The project involves 31 works characterized in Table 1 .

Figure 3 shows a network (graph) project work ordering model where arrows indicate works, circles indicate the beginning and end of work, dashed arrows show dummy operations needed to demonstrate the relations among works. 


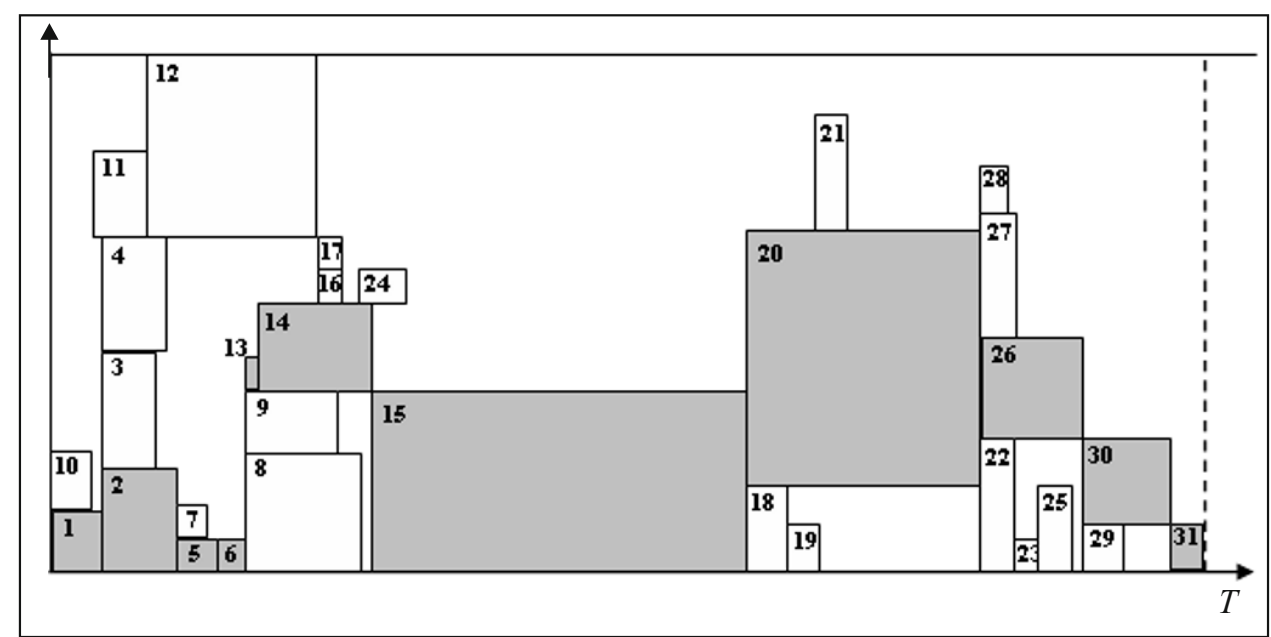

Fig. 4. Determining the critical path of the project (critical operations are grayed).

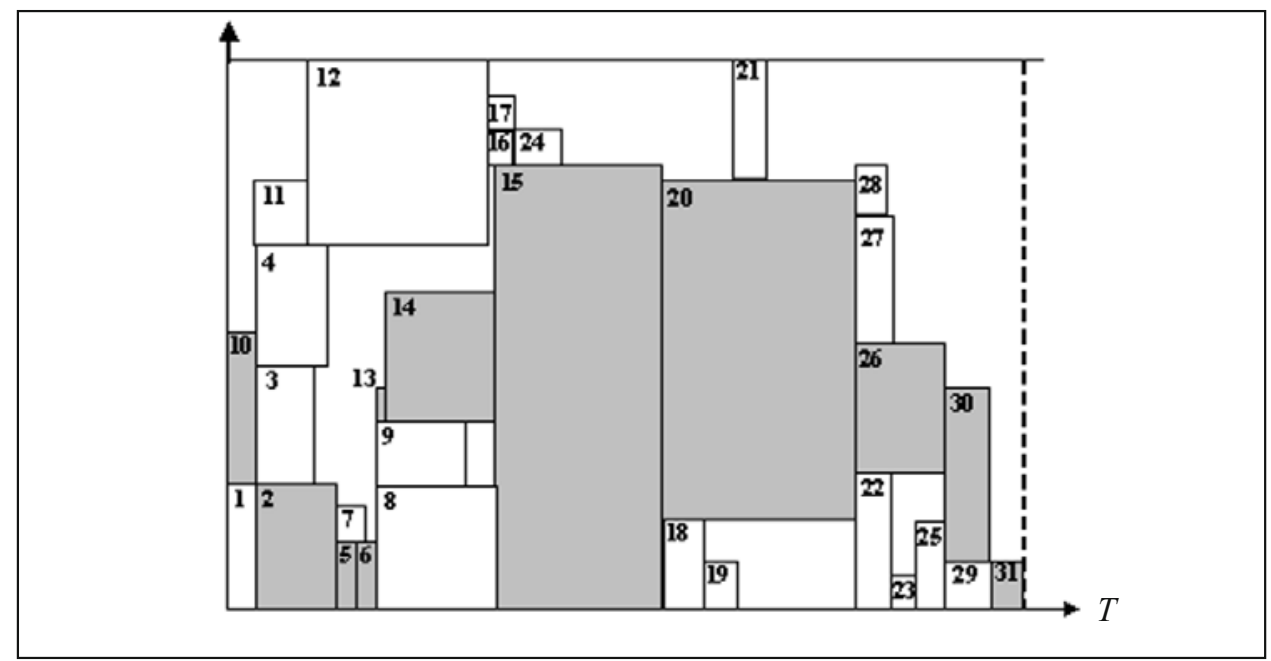

Fig. 5. Optimization of characteristics of critical operations.

By solving problem (9), we have estimated the necessary number of technical experts (18) engaged in the project and the critical path of the project (145 days; Fig. 4). Solving problem (13) made it possible, by intensifying the critical works without affecting the characteristics of noncritical operations and increasing the number of workers, to reduce the project time to 81 days (Fig. 5).

\section{CONCLUSIONS}

We have constructed a mathematical model of the multicriterion problem of the allocation of limited resources of a project as a set of separable optimization problems of the allocation of a finite set of geometrical objects with variable metric characteristics. We have emphasized the main properties of the problem and considered the exact and approximate methods of its solution. We have also proposed an approach to constructing a linear approximation of separable functions of problem constraints, which differs from the well-known approaches in the possibility of specifying the approximation accuracy as an exogenous parameter, and also ensuring the membership of the approximation polyhedral set in the original space of independent variables of the problem. We have performed an algorithmic and program implementation of the proposed methods and solved the practical problem of determining the parameters of the project of an automatic fire alarm 
system. The further studies imply the construction of the computational algorithms of the solution of $3 D$ packing problems for objects with variable metric characteristics.

\section{REFERENCES}

1. E. J. Umble, R. R. Haft, and M. M. Umble, "Enterprise resource planning: Implementation procedures and critical success factors," Europ. J. Oper. Res., No. 146, 241-257 (2003).

2. I. I. Masur and V. D. Shapiro (eds.), Project Management: A Reference Book [in Russian], Vyssh. Shk., Moscow (2000).

3. V. V. Kryzhanovskyy and S. N. Popov, "Integrated project management system," Cybern. Syst. Analysis, 45, No. 6, 966-970 (2009).

4. A. N. Voronin, "A multicriteria problem of distribution of bounded resources," Cybern. Syst. Analysis, 47, No. 3, 490-493 (2011).

5. Zh. He, R. Liu, and T. Jia, "Metaheuristics for multi-mode capital-constrained project payment scheduling," Europ. J. Oper. Res., No. 223, 605-613 (2012).

6. M. Mika, G. Waligyra, J. Weglarz, "Tabu search for multi-mode resource-constrained project scheduling with schedule-dependent setup times,” Europ. J. Oper. Res., No. 187 (3), 1238-1250 (2008).

7. V. S. Mikhalevich and V. V. Shkurba, "Sequential optimization methods in problems of operation scheduling," Cybernetics, 2, No. 2, 28-33 (1966).

8. T. P. Podchasova, V. M. Portugal, V. A. Tatarov, and V. V. Shkurba, Heuristic Scheduling Methods [in Russian], Tekhnika, Kyiv (1980).

9. A. I. Slyeptsov and T. A. Tyshchuk, "A method of computation of characteristics of operation in a problem of fuzzy network planning and management," Cybern. Syst. Analysis, 39, No. 3, 367-378 (2003).

10. M. V. Novozhilov and T. E. Romanova, "The factor of uncertainty of the time parameter in project management," Probl. Mashinostr., 4, No. 1-2, 79-84 (2001).

11. B. R. Sarker, P. J. Egbelu, T. W. Liao, and Y. Junfang, "Planning and design models for construction industry: A critical survey," Automation in Construction, No. 22, 123-134 (2012).

12. E. G. Petrov, M. V. Novozhilova, and I. V. Grebennik, Methods and Tools of Decision Making in Socio-Economic Systems [in Ukrainian], Tekhnika, Kyiv (2003).

13. Kh. A. Takha, An Introduction to Operations Research [in Russian], Vil'yams, Moscow (2001).

14. I. A. Chub and M. V. Novozhilova, "Analytical description of the condition of the membership of an object with variable metric characteristics in the domain of layout," Systemy Obrobky Informatsii, Issue 6 (22), 248-252 (2002).

15. M. V. Novozhilova and N. O. Popelnyukh, "Solving the optimization problem for project resources under exact initial data," Visn. ZhDTU, Tekhn. Nauky, No. 4 (39), 225-230 (2006).

16. Yu. G. Stoyan and S. V. Yakovlev, Mathematical Models and Optimization Methods of Geometrical Design [in Russian], Naukova Dumka, Kyiv (1986).

17. I. A. Chub, "Geometric modeling of basic constraints for the parameters of the arrangement of objects wit variable metric characteristics,” Prykl. Geom. Inzh. Grafika, Zb. Nauk. Prats', TDATU, Melitopol, 42, Issue 4, 77-85 (2009).

18. I. A. Chub, M. N. Murin, and M. V. Novozhilova, "A method to solve the problem of arrangement of rectangles with variable metric characteristics," Radioelektronika i Informatika, No. 4, 134-141 (2007).

19. F. Hill, W. Murray, and M. Right, Practical Optimization, Mir, Moscow (1985).

20. M. R. Garey and D. S. Johnson, Computers and Intractability: A Guide to the Theory of NP-Completeness, Freeman, San-Francisco (1979).

21. A. A. Lipnitskii, "Use of genetic algorithms for solution of the rectangle packing problem," Cybern. Syst. Analysis, 38, No. 6, 943-946 (2002).

22. I. A. Chub and M. V. Novozhilova, "The finite method of searching for the global minimum in the problem of arrangement of rectangular objects," Dop. NANU, No. 11, 56-61 (2011). 